\title{
RIFLESSIONI SULLA METODOLOGIA DEGLI STUDI COMPARATI
}

di Neil J. Smelser

Sono passati vent'anni da quando pubblicai Comparative Methods in the Social Sciences (Smelser 1976). Il libro riscosse un certo interesse da parte della sociologia e della scienza politica. Tuttavia, dovrei mettere in chiaro che la sua pubblicazione $\mathrm{fu}$ in qualche modo singolare. Nella mia carriera non mi sono mai considerato un metodologo, malgrado in alcuni periodi abbia insegnato metodologia. $E$ benché ritenga che il messaggio di quel volume sia congruente con gran parte della mia ricerca sostantiva, avevo scritto ben poco sui metodi di comparazione prima dell'uscita del libro, e niente ho scritto sul tema in seguito. L'invito a tenere una lezione presso l'Istituto Universitario Europeo $\mathrm{mi}$ ha offerto una gradita opportunità di passare in rassegna alcuni sviluppi occorsi dopo la seconda metà degli anni Settanta e di elaborare nuove riflessioni su alcune tematiche metodologiche nelle scienze sociali comparatistiche. Queste mie riflessioni si svilupperanno in quattro parti: a) alcune informazioni sui precedenti della stesura del libro e un'analisi retrospettiva dei suoi contenuti e dei suoi scopi intellettuali; $b$ ) una rassegna dei contributi alla letteratura sulla metodologia comparatista che sono apparsi in seguito; $c$ ) un tentativo di mettere ordine in questa letteratura confusa; $d$ ) una breve riconsiderazione finale di un paio di problemi centrali che verranno allo scoperto in questa presentazione.

Comparative Methods $e$ il suo retroterra

Per quanto ricordi, il mio interesse per i metodi di analisi comparata nacque negli anni 1952-54 a Oxford, dove ero un

Questo articolo riprende il testo di una lezione tenuta presso l'Istituto Universitario Europeo il 2 giugno 1995.

RIVISTA ITALIANA DI SCIENZA POLITICA / a. XXVI, n. 1, aprile 1996 
semplice studente universitario, quando mi accorsi che non mi andavano a genio la faciloneria e il pressappochismo con cui i miei colleghi studenti - molti stranieri - erano disposti a generalizzare circa il carattere e le istituzioni di altri paesi. Feci persino una breve presentazione in un club intellettuale di cui ero membro sui pericoli della costruzione di stereotipi nazionali. Questa insofferenza si rinnovò negli anni del mio dottorato a Harvard, ove era allora in voga la letteratura sul «carattere nazionale» - a volte ricca di intuizioni ma per lo più insoddisfacente.

Nei primi tempi della mia carriera accademica a Berkeley non coltivai questo atteggiamento di insoddisfazione latente, ma l'interazione con comparatisti eminenti quali Reinhard Bendix, Ernst Haas, David Apter e Seymour M. Lipset, come pure gli sviluppi delle mie attività di ricerca empirica, riaccesero l'interesse. Infatti, presi l'iniziativa - nel 1964 o nel 1965, mi pare di avvicinare Lipset per proporgli di collaborare a una monografia sulla metodologia dell'analisi comparata. Lipset - con cui avevo già collaborato - si dimostrò ben disposto, sicché abbozzai per iscritto alcune idee preliminari. Tuttavia, Lipset non dette seguito alla cosa; da lì a un paio d'anni si spostò a Harvard. Alla fine degli anni Sessanta decisi che, in qualche modo, sarei andato avanti da solo. Scrissi dei saggi iniziali che vennero pubblicati (Smelser 1967; 1971; 1973), dopodiché approfittai di un anno sabbatico in Europa nel 1973-74 per preparare l'intera monografia.

I contenuti del libro si svilupparono nel modo seguente. All'inizio presi in considerazione Tocqueville come comparatista (principalmente della Francia, degli Stati Uniti e della Gran Bretagna, anche se i suoi lavori trattano anche altri casi). Sostenni che Tocqueville poneva attenzione a un insieme specifico di problemi sostantivi (e cioè l'avanzare della democrazia e l'eclissi del feudalesimo), ma soprattutto che, malgrado il carattere saggistico e informale della maggior parte della sua opera, le sue argomentazioni riguardo le differenze fra questi paesi obbedivano ai canoni di tenere costanti e variare le condizioni storiche nello sforzo di corroborare le spiegazioni che prediligeva. Poi trattai altri due giganti della tradizione comparatista, Emile Durkheim e Max Weber, i cui principi epistemologici e metodologici sono agli antipodi, ma le cui ricerche empiriche mostrano un modello affine di manipolazione concettuale ed empirica delle informazioni statistiche e storiche per escludere certe 
determinanti storiche e fissarne altre. Il tentativo era rendere evidente che, in fondo, il lavoro empirico di questi due studiosi ha caratteristiche metodologicamente comuni e coerenti.

Il resto del volume consisteva in un esame formale delle problematiche principali che si incontrano nella comparazione tra casi (cross-unit comparison) - che possono essere nazioni o altre unità - i quali sono chiaramente dissimili fra loro sotto molti aspetti. Queste problematiche erano: la classificazione delle unità (che comprende la questione della loro comparabilità), la descrizione delle informazioni riguardanti queste unità, la misurazione (che comprende la questione della comparabilità degli indicatori); e, nel capitolo finale, i problemi dell'associazione, della causa, della spiegazione e dell'interpretazione teorica. I problemi discussi e le argomentazioni sollevate nella seconda parte di questo volume venivano illustrate sulla base di esempi di ricerche comparate contemporanee in scienza politi$\mathrm{ca}$, sociologia, antropologia ed economia.

Il libro era sintetico. Intendeva rendere conto dell'insieme delle modalità del fare ricerca e interpretazione, ma all'interno di una cornice comune. Le modalità di ricerca a cui faceva riferimento erano la sperimentazione, la manipolazione statistica, l'illustrazione comparata sistematica quando il numero dei casi è limitato (alcuni chiamano proprio questa operazione «analisi comparata»), l'analisi dei casi devianti, gli assunti caeteris paribus e l'«esperimento immaginario» (o argomentazione controfattuale). Tentavo di dimostrare che tutte queste modalità sono simili sotto un profilo: tutte - e tutti i ricercatori che le impiegano - «cercano di controllare e manipolare varie condizioni causali nella vita sociale [tenendole costanti e variandole], e di provare così l'influenza di una o un'altra condizione» (Smelser 1976, 5). Questa era la cornice comune. Al suo interno cercavo di valutare ciascuna modalità di ricerca nei termini del tipo di controllo che può consentire di ottenere, delle specifiche difficoltà che implica, e della fiducia nei risultati che si possono conseguire (o meno) con essa. Ne risultò un libro di metodologia generale, e forse non avrei dovuto riferirmi ai «metodi di comparazione» in particolare; ma rappresentava uno sforzo di inserire in una singola prospettiva di metodo-ricerca l'analisi comparata di più paesi (cross-national) in sociologia, scienza politica e antropologia. Il lavoro traeva la maggior parte dei suoi esempi da queste aree di ricerca e venne letto soprattutto da studiosi che si riconoscevano in queste tradizioni disciplinari. 
Il libro era sintetico anche in un altro senso, per il fatto che cercava di collocarsi a metà strada fra due famiglie di punti di vista nelle scienze sociali. Da un lato rigettava l'approccio positivista radicale, secondo cui si possono comparare direttamente misure comuni e oggettive (indicatori economici, risposte a sondaggi di opinione) in diversi contesti e «scoprire» in questo modo regolarità e leggi generali. Nello stesso senso, ribadivo l'importanza dei contesti nazionali e culturali nella ricerca e nell'interpretazione dei dati empirici. Dall'altro lato, rigettavo anche la posizione relativista secondo cui il contesto è tutto e quindi, in ultima analisi, nessun oggetto può essere comparato in quanto incastonato in un contesto unico, sicché non si può ottenere alcuna conoscenza generale. Questa tensione fra positivismo e relativismo è una versione di un conflitto generale che si può ritrovare in altre polarità nelle scienze sociali e del comportamento: universalizzare contro particolarizzare (o storicizzare) la conoscenza, modalità nomotetiche contro idiografiche, e - in alcune discussioni - ricerca sociologica contro ricerca storica.

Il mio sforzo mirava a riconoscere l'importanza degli aspetti contestuali per generare misure e spiegazioni comparate, ma anche a cercare di identificare strategie feconde per prendere in considerazione quel contesto, in modo da migliorare l'analisi comparata e tentare di sospingere la conoscenza al livello più generale possibile.

Un indicatore del carattere sintetico di questo sforzo si trova nelle critiche che ho ricevuto. Feci precedere la pubblicazione del libro dalla diffusione del manoscritto all'interno di un simposio che presiedevo nella mia facoltà. Il gruppo si chiamava, abbastanza appropriatamente, «Gruppo di teoria e metodo degli studi comparati» e aveva sede nell'Istituto di studi internazionali di Berkeley. Il manoscritto venne criticato da Reinhard Bendix per i suoi assunti positivisti e da Arthur Stinchcombe e Charles Glock perché la dava vinta alla scienza sociale non scientifica e interpretativa. Temo che questa sia la sorte di chi cerca di promuovere la pace.

\section{Le prese di posizione successive}

Diversi anni dopo la pubblicazione di Comparative Methods in the Social Sciences, Theda Skocpol e Margaret Somers 
(1980) scrissero un articolo dedicato specificamente alla storia comparata, ma con riferimenti di portata più generale, in cui criticavano il mio lavoro, come quello di altri, per avere «cercato erroneamente di far rientrare tipi distinti di storia comparata in un'unica logica metodologica» (ibidem, 175). (Questa questione, sia detto per inciso, si rivela essere un problema molto generale in buona parte delle discussioni metodologiche degli ultimi due decenni). Per contro, Skocpol e Somers identificavano tre logiche «distinte»: a) l'analisi macrocausale, che indicavano come «la verifica di un'ipotesi multivariata»; b) la dimostrazione parallela di una teoria, o ricerca di modelli comuni; c) i «contrasti di contesti», o l'accentuazione della non comparabilità di casi discreti. Esse sviluppavano diversi esempi di ciascuno di questi tipi nella letteratura comparata contemporanea. Per quanto utile per certi scopi, forse, questa classificazione soffre del fatto che i tre tipi non sono in effetti «distinti»; sembrano, piuttosto, disporsi lungo un continuum dal generalizzante al particolarizzante e implicare pretese causali che, per di più, possono essere tutte verificate secondo gli stessi canoni metodologici. Oltre a questa osservazione critica, tuttavia, mi sia concesso notare che per Skocpol e Somers un importante stimolo polemico è costituito dalla volontà di determinare l'integrità di un'entità o metodo detto «storia comparata».

Diversi anni dopo Charles Ragin (1987) ha scritto un trattato più ambizioso sul metodo comparato. Uno degli obiettivi polemici del suo lavoro è il mio libro, ma l'obiettivo è perseguito seguendo una pista differente, per cui io sarei responsabile di avere degradato lo studio del caso comparato ad una sorta di cittadino metodologico di seconda classe:

Smelser sostiene che ... il metodo dell'illustrazione comparata sistematica è inferiore al metodo statistico come metodo di comparazione. $\grave{E}$ inferiore, secondo Smelser, perché deve essere impiegato quando il numero di casi rilevanti è piccolo e la possibilità di ottenere un controllo sistematico sulle fonti di variazione dei fenomeni sociali è ridotto ... Smelser sostiene che un metodo comparato rigoroso è una contraddizione in termini (ibidem, 12 e 13).

In una presa di posizione precedente, Ragin aveva affermato, contro i critici del mio lavoro, che il metodo comparato «non è un cugino bastardo del metodo statistico» $(1981,113)$.

Sforzandosi di elevare quello che avevo chiamato «metodo comparato» a uno status scientifico più adeguato di quanto egli 
ritiene io abbia fatto, Ragin è arrivato a dire che esso è superio$r e$ al metodo statistico per quattro aspetti: il metodo statistico non è combinatorio; il metodo comparato tiene conto di ogni accadimento di un dato fenomeno; il ricercatore comparatista non deve fingere di disporre di un campione di società tratto da una determinata popolazione; il metodo comparato obbliga il ricercatore a familiarizzarsi con i casi rilevanti per la sua analisi. A mio modo di vedere, solo quest'ultimo è un vantaggio; quanto agli altri, Ragin definisce come punti di forza quelle che in effetti sembrano essere specifiche difficoltà che emergono quando si ha a che fare con piccoli campioni tratti da una popolazione ignota. Sia come sia, Ragin è giunto a sviluppare una tecnica speciale basata sull'algebra booleana per semplificare complesse strutture di dati. La mia lettura dell'esposizione di Ragin pare rivelare che il suo obiettivo di fondo è di elevare una certa entità - il metodo dello studio del caso comparato ad una posizione specifica e con eguale rango degli altri metodi di ricerca nell'arsenale dello scienziato sociale.

Uno degli «assunti metateorici» su cui Ragin diceva di basarsi era che «le società esistono». Nel frattempo, Immanuel Wallerstein e altri avevano lanciato una critica molto più radicale alle linee di ricerca cross-national, cross-cultural e cross-societal, sfidando proprio quell'assunto. Uno dei punti polemici centrali della teoria della dipendenza (Frank 1971) e dell'analisi del sistema-mondo (Wallerstein 1974) era che la «teoria della modernizzazione» degli anni Cinquanta e Sessanta aveva trattato erroneamente ogni società come un'unità indipendente e lo sviluppo come una variabile esogena. Questo approccio, sostenevano, ignorava i modelli di dominazione internazionale passati e attuali esercitati da parte delle nazioni e delle economie più forti (centrali) su quelle più deboli (periferiche). Wallerstein $(1978 ; 1984)$ e altri (per esempio, Bach 1977) hanno sollevato il punto in chiave metodologica, ritenendo che, in effetti, quell'analisi comparata (la comparazione sistematica delle nazioni) non ha senso dato che le nazioni non sono affatto unità (certamente non unità indipendenti); l'unità d'analisi è piuttosto il sistema - il sistema-mondo per l'esattezza - e le storie e gli sviluppi nazionali sono subordinati alle dinamiche di quel sistema. Alla luce di questo asserto, lo studio delle società richiede metodologie interamente nuove (per esempio, la «comparazione incorporata» di McMichael: 1990), diverse dalle strategie di comparazione basate sull'idea erronea, e da positivisti ottocen- 
teschi, secondo cui le nazioni sono unità sovrane e indipendenti che possono essere trattate con metodi di analisi adatti a questo assunto (per esempio, la comparazione delle correlazioni interne di certi fenomeni).

Sempre a metà degli anni Ottanta, Charles Tilly (1984) ha prodotto il suo manifesto sulla comparazione, che rappresenta il culmine di una lunga e onorata carriera di ricerca in storia politica comparata. Tilly comincia il suo lavoro come una critica in gran parte sostantiva di quelli che chiama «otto postulati perniciosi» del pensiero del secolo XX risultanti da una lettura errata del secolo XIX. La sua prima critica, di portata metodologica simile alle critiche delle teorie della dipendenza e del sistema-mondo, attacca il postulato per cui «la società è una cosa a sé», secondo il quale le società sono unità distinte «che hanno proprie culture, governi, economie e solidarietà autonome» ( $i b i$ dem, 11). Fra gli altri postulati che Tilly rigetta vi sono la teoria degli stadi del cambiamento sociale, la logica della differenziazione, l'interpretazione dello stato dell'ordine sociale come un effetto dell'equilibrio di differenziazione e integrazione, dei problemi sociali come frutto di tensioni derivanti da un cambiamento sociale troppo rapido, e la distinzione fra forme di conflitto «illegittime» e «legittime». La polemica principale di Tilly si rivolge, evidentemente, contro la tradizione funzionalista di Parsons e di altri, collocandosi nel filone dagli incerti contorni della «sociologia del conflitto». Anche da un punto di vista sostantivo, egli ritiene che le più importanti dinamiche di cambiamento risultano dall'avanzata del capitalismo (che comprende $i$ fenomeni dell'urbanizzazione, della crescita della popolazione e della proletarizzazione) e dallo sviluppo di «stati nazionali vasti e connessi tra loro» (ibidem, 15). (Ma il riferimento agli «stati nazionali», indicati anche come «unità politiche relativamente indipendenti», non è un modo di reintrodurre un livello di analisi parallelo alla società?). Con ciò, questa sembra essere la principale posizione sostantiva di Tilly.

Da un punto di vista metodologico, Tilly ha un atteggiamento critico anche verso gli studi che «mettono insieme un ampio numero di casi in analisi statistiche di carattere estensivo» e verso le comparazioni fra casi nazionali aggregati, a cui preferisce «attente comparazioni di piccoli numeri» (ibidem, 76-77). E mentre afferma di non voler entrare in una vera «discussione della logica della comparazione in quanto tale» (ibidem, 15), identifica comunque un certo numero di «strategie per la com- 
parazione di grandi strutture e vasti processi». Queste strategie, tuttavia, producono una tipologia relativamente semplice e formale di modalità di individualizzazione, inclusione, universalizzazione e ricerca della variazione. La tipologia è derivata da due dimensioni: la quota complessiva di esempi comparati compresi nell'analisi e la molteplicità delle forme considerate. Nel suo libro, Tilly illustra come è trattata ciascuna strategia nella letteratura contemporanea e conclude che tutte hanno qualcosa da offrire e «posti sicuri nel nostro strumentario intellettuale» (ibidem, 147). Come lui stesso confessa, questa tipologia, paragonata con ogni altra logica formale di comparazione, è «poco conveniente» (ibidem, 144).

La proposta di Tilly suscita immediatamente due critiche: a) non c'è un legame fra la sua critica sostantiva della teoria funzionalista del cambiamento sociale e i suoi tipi metodologici; i secondi non derivano in alcun modo dalla prima, e avrebbero pure potuto essere inclusi in una pubblicazione a parte; $b$ ) la discussione metodologica porta a una classificazione di stili di pensiero, ma non affronta nessun problema metodologico formale quale la classificazione, l'inferenza e la spiegazione. Ci si può chiedere - l'ho fatto io stesso - perché Tilly l'abbia inserita in una discussione di metodologia della comparazione. E una buona domanda, a cui rispondo che questa prospettiva non trova posto nel più ampio dialogo degli ultimi vent'anni, come spiegherò adesso.

Mi riferisco a un ultimo contributo - molto recente - di tre scienziati politici, Gary King, Robert Kehoane e Sidney Verba (1994). Il loro libro è soprattutto un manuale di metodologia. Esso descrive la logica fondamentale dell'analisi statistica multivariata oggi in voga e sostiene che questa logica e queste procedure possono essere generalizzate in modo da comprendere la ricerca qualitativa e da migliorare, quindi, il livello di questo tipo di ricerca. La ricerca qualitativa è definita formalmente come quella ricerca «in cui la misurazione numerica è o impossibile o non desiderabile» (ibidem, IX), ma gli autori hanno anche in mente la ricerca basata su pochi casi oppure su metodi etnografici intensivi e analisi di dati d'archivio o, ancora, su argomentazioni discorsive (ibidem, 4); dunque, si usa «qualitativo» in una maniera piuttosto imprecisa, come spesso succede nelle scienze sociali. La tesi centrale del libro è che vi è una «logica unificata dell'inferenza» che si applica a tutta la ricerca so- 
ciale e che la ricerca qualitativa ha molto da guadagnare laddove impieghi questa logica nel modo più rigoroso. Il contenuto del volume comprende parti sulla falsificabilità, sulla misurazione, sulla congruenza interna dei modelli, sul campionamento, sulla distorsione nella selezione dei casi (selection bias), sulle strategie per l'ampliamento del numero dei casi e su altri argomenti che rientrano nel metodo scientifico così come è inteso specificamente nel lavoro delle scienze sociali.

Si è criticato il testo per il suo tono catechizzante (Brady 1995), per la sua eccessiva attenzione a concezioni piuttosto limitate dello sperimentalismo e della causalità (Caporaso 1995), per la sua insufficiente attenzione per la teoria (Rogowski 1995). Nel complesso, esso si segnala come un tentativo di conciliazione (fra approcci dagli accenti quantitativi e qualitativi), sebbene giunga a ciò tramite una generalizzazione ed estensione dell'approccio quantitativo ed una vigorosa riasserzione dei canoni della scienza positiva. Questa riasserzione, tuttavia, viene fatta alla fine di un lungo periodo di attacco a questi metodi da parte di studiosi marxisti, teorici critici, fenomenologi, decostruzionisti e filosofi della scienza.

\section{Una disamina della letteratura}

Si possono fare poche osservazioni generali sulla letteratura che ho citato. Per prima cosa, questa letteratura costituisce soprattutto una versione complessa e a volte confusa dell'annoso dibattito sullo status delle scienze sociali positive. Incidentalmente, potrei dire che se si vuole compiere una lettura complessiva della storia delle scienze sociali (specialmente in Ameri$\mathrm{ca}$, ma anche in Europa), è opportuno fare i conti con l'influenza enorme e forse sovrastante esercitata dalla visione che si ha dell'immagine della «scienza» sull'organizzazione e la legittimazione dell'attività di ricerca e sulla definizione dei dibattiti fondamentali in questo campo. Quanto segue è la spiegazione di un piccolo episodio che si integra in questo quadro più ampio.

Si può scomporre il dibattito in componenti - o sottospecie - della letteratura passata in rassegna.

Si scopre, in primo luogo, un tipo di dibattito sul fatto se vi sia una unità o una diversità di metodi - o, più precisamente, logiche di inferenza - nell'analisi comparata. Su questo punto, 
King, Kehoane e Verba occupano l'estremo dell'«unità»; io mi schiero dalla loro parte su questo aspetto; Ragin e Skocpol condividono invece il punto di vista della «diversità». Tilly non prende posizione sulla «logica dell'inferenza» - anche se è profondamente critico per quanto riguarda le comparazioni statistiche aggregate fra nazioni - ma identifica un insieme distinto di «strategie». La posizione di Wallerstein consiste nel rifiutare del tutto le basi tradizionali della comparazione fra nazioni, sostituendovi la nuova logica del sistema-mondo, in cui le dinamiche complessive sono ricondotte agli sviluppi storici dei poteri nazionali. $\mathrm{Ci}$ si può chiedere - come io stesso mi sono chiesto perché il problema unità-diversità debba generare tanta vis polemica, quando invero non sembra essere una questione molto rilevante. La mia opinione è che ci troviamo di fronte a due tipi generali di discorso in questi scambi polemici. Il primo riguarda l'unità stessa della scienza. Fra le correnti più utopistiche del positivismo, ritenere che la scienza sia un tutto unificato rappresenta una posizione teorica prevalente da molto tempo (questa fede ha forse la sua ultima origine nelle aspettative utopistiche prodotte dalla fisica newtoniana). Altri hanno invece sostenuto che lo studio degli esseri umani e della società richiedano una specie differente di conoscenza. Non c'è dubbio che Max Weber ebbe particolarmente a che fare con questo dibattito nei suoi sforzi di elaborare una sintesi tra il positivismo anglosassone e lo storicismo tedesco dei suoi tempi.

Il secondo, e forse più importante, conflitto che soggiace a questo dibattito ha un carattere più «parrocchiale», consistendo in una sorta di disputa giurisdizionale fra metodologi. Una prova decisiva di ciò è l'attacco sferrato da Ragin contro il mio approccio dell'«unità dell'inferenza», ritenuto colpevole di degradare o addirittura di eliminare il metodo della ricerca comparata (meglio inteso come illustrazione comparata sistematica di un numero limitato di nazioni o società per giungere a una conoscenza esplicativa). Per lo più la polemica di Ragin nei miei confronti, così come le sue argomentazioni e formulazioni in positivo, mira a nobilitare quell'entità riconoscibile, l'analisi comparata, e a nobilitarla proclamandone il carattere scientifico - anzi, più scientifico in qualche modo dei metodi sperimentale e statistico. La posizione di Skocpol è simile, in quanto giunge a identificare una serie di metodi distinti di ricerca comparata oltre ai metodi macrocausali o multivariati. L'immagine di 
scienza è anche l'obiettivo critico di Tilly e Wallerstein, il cui lavoro indica che i metodi di comparazione basati su presupposti positivisti sono illegittimi perché riflettono la Weltanschauung erronea delle scienze sociali positiviste. Alla luce di queste posizioni, credo che si possa apprezzare la mia precedente osservazione circa l'importanza dell'ombra del positivismo in molte dispute delle scienze sociali.

Per inciso, questo dibattito sulla legittimità ed integrità di diversi metodi di ricerca echeggia i dibattiti sui programmi d'insegnamento nei dottorati di sociologia in America. Venti o trent'anni fa, il modello dominante consisteva nel richiedere a tutti i dottorandi di seguire corsi di metodologia quantitativa, corsi in cui si trattavano temi quali il disegno della ricerca, la codifica, il campionamento e le tecniche statistiche. Col tempo, però, i sostenitori di «altri» metodi hanno cominciato a difendere sia la legittimità dei metodi di comparazione, di ricerca d'archivio ed etnografica che la richiesta di un «egual numero di ore» di insegnamento per questi metodi. Le soluzioni al dibattito nella pratica dei programmi di insegnamento sono state miste. In alcune università rimane il predominio dei metodi quantitativi; in altre, accanto al corso obbligatorio di metodologia quantitativa, viene offerto un corso obbligatorio su aspetti più generali della ricerca, che include temi di filosofia della scienza. In altri casi ancora, si sono individuati una serie di corsi di metodologia «opzionali» su queste tematiche. In seguito a tali controversie ci si è spostati generalmente in direzione di un maggior «pluralismo»; tale spostamento - insieme ad altri sviluppi in questo campo - fa sì che la sociologia sembri diventare sempre più frammentata. Si potrebbero citare sviluppi analoghi, seppure diversi per accenti e tono, anche in altre scienze sociali.

Uno degli aspetti più frustranti della letteratura è la fusione della questione della logica dell'inferenza negli studi comparati con l'opposizione - annosa, sfaccettata e molto caotica - fra ricerca quantitativa e qualitativa. Questa confusione si ritrova sia nel lavoro di Ragin che in quello di King, Kehoane e Verba. In gran parte anche questo dibattito ha un carattere giurisdizionale, e non è difficile capire perché assomigli al precedente. La mia opinione è che, quale che sia il significato - uno fra i molti possibili - assegnato ai termini «quantitativo» e «qualitativo», questa assegnazione ha poco a che vedere con la spiegazione e la comprensione di regole e metodi da applicare nei canoni dedut- 
tivi e inferenziali che collegano la teoria scientifico-sociale e la ricerca empirica. Se questi due dibattiti fossero separati, se ne guadagnerebbe non poco.

Un altro aspetto della letteratura che crea confusione è la sovrapposizione di prospettive sostantive e questioni metodologiche. In piccola parte questa sovrapposizione si trova nel termine che usa Skocpol, «analisi macrocausale», come se vi fosse una specie distinta di analisi causale nell'analisi delle strutture macro. La confusione è ancora più evidente nel lavoro di Tilly. Per la maggior parte la sua polemica ha un carattere sostantivo e teorico, in antitesi con gli assunti e gli accenti dell'approccio struttural-funzionalista in sociologia. Questa polemica si collega tangenzialmente alla sua opposizione ai metodi statistici aggregati, ma quasi per niente ai suoi quattro «metodi» o «strategie» o «stili» di ricerca comparata. A mio modo di vedere, se la scelta dei metodi e delle strategie di ricerca è connessa agli interessi teorici del ricercatore, è possibile discutere la logica per determinare l'adeguatezza delle spiegazioni senza far riferimento ai contenuti sostantivi della ricerca. Perfino coloro che, come Wallerstein, propongono di accantonare buona parte della ricerca comparata in ragione della non-indipendenza delle unità nazionali, non possono fare a meno di affrontare la logica dell'inferenza e della spiegazione quando avanzano asserti empirici sui processi che hanno luogo nel sistema-mondo.

Vi è, infine, una sorta di dimensione politica peculiare nei dibattiti recenti. Durante gran parte della sua storia, la scienza positiva ha avuto un connotato radicale e rivoluzionario, per lo più dovuto agli attacchi che portava a posizioni tradizionali dominanti in teologia, morale e filosofia (vengono in mente le figure di Copernico, Galileo, Darwin e Freud). Negli ultimi decenni, tuttavia, c'è stato un rovesciamento - parlo solo delle scienze sociali, e c'è differenza al loro interno - con la scienza positiva che rappresentava una specie di ortodossia, una specie di destra intellettuale se si vuole, mentre gli attacchi radicali provenivano dalle fonti non-positiviste che ho menzionato prima, ossia il neo-marxismo, l'umanismo, la fenomenologia e il decostruzionismo. $\dot{E}$ anche interessante osservare, e mi preme sottolinearlo, che questa dimensione destra-sinistra intellettuale è correlata molto blandamente con la dimensione politica destra-sinistra ereditata dal passato; cioè, chi aderisce alla prospettiva della scienza sociale positiva tende ad avere un atteggiamento meno critico degli altri verso la società post-industriale 
contemporanea. Non dovremmo dare troppo peso agli aspetti politici di questi dibattiti, ma essi sono abbastanza scoperti da doverne fare menzione e da notare che formano un altro ingrediente del complesso di questioni che compongono i dibattiti metodologici, sì da renderli più confusi ma anche meno aridi di quanto ci si potrebbe altrimenti aspettare.

\section{Alcune riflessioni conclusive}

Vi sono quattro riflessioni che vorrei aggiungere su ciò che ho esaminato fin qui:

1) Nei due decenni di discussione che ho preso in considerazione si è potuta osservare una dialettica interessante. La pubblicazione del mio libro giungeva al culmine di un piccolo boom della letteratura politologica e sociologica volta ad affinare la metodologia comparatista all'interno della tradizione che si può approssimativamente definire «positivista» (per esempio, Sartori 1970; Przeworski e Teune 1970; Vallier 1971; Lijphart 1971; Armer e Grimshaw 1973). Se me lo chiedessero, collocherei il mio libro in questa stessa tradizione, nonostante mi sia sforzato in modo particolare di affrontare le concezioni «relativistiche» del contesto culturale e di altri problemi. Nei due decenni successivi si è scatenata un'aspra diatriba in merito alle problematiche dell'integrità e dello status scientifico del «metodo comparato» - una diatriba che rifletteva movimenti di più ampia scala nelle scienze sociali. La pubblicazione di Designing Social Inquiry segna una riaffermazione estremamente energica della logica di fondo dell'analisi quantitativa e multivariata. Non è difficile prevedere che questa pubblicazione provocherà uno scroscio di proclami e prese di posizione proscientifiche e antiscientifiche; probabilmente, assisteremo a una certa ripetizione dei temi che abbiamo osservato negli ultimi vent'anni. A causa della nostra persistente preoccupazione per l'immagine della scienza, tendiamo a riprodurre dialoghi e dispute del passato - in una specie di compulsione a ripetere - che non necessariamente aggiungono molto alla storia delle nostre discipline.

2) Come ho suggerito, credo sia possibile separare le discussioni sulla logica dell'inferenza nelle scienze sociali dagli sviluppi teorici e, se posso prendere un tono esortativo al riguardo, penso che si percorrerebbe un bel tratto di strada intellettuale se si effettuasse questa separazione. Non voglio dire che 
la teoria sostantiva e la filosofia della scienza non sono legate alla metodologia e non dovrebbero essere coltivate. Tuttavia, vorrei sostenere che le discussioni metodologiche possono essere sviluppate per conto proprio e secondo la propria logica.

3) Analogamente, ritengo anche che sia possibile separare le discussioni generali di metodologia dalla sostanza della ricerca. Non c'è una logica distinta che entra in gioco nello studio comparato del linguaggio, della formazione di coalizioni politiche, di strutture sociali o di valori culturali. Inoltre, penso che sia difficile per coloro che propongono una nuova prospettiva teorica in competizione con le precedenti esimersi dall'osservare i canoni correnti di evidenza, inferenza, causa e spiegazione. Se vogliono esimersi dalle scienze sociali tout court, o affermare che la spiegazione scientifico-sociale è impossibile, è un altro discorso. Ma vorrei sostenere che finché manteniamo un impegno generale a portare avanti l'impresa scientifica, assumiamo anche un impegno ad osservare quei canoni metodologici e ad essere criticati sulla loro base.

4) La revisione metodologica più importante suggerita dalla letteratura proviene dalla prospettiva d'analisi del sistema-mondo. La sfida di Wallerstein all'analisi comparata non è nuova. Quasi un secolo fa, il problema dell'indipendenza delle unità venne sollevato come «il problema di Galton» - la cui sostanza è che se le unità si sono influenzate in qualche modo (ad esempio, per diffusione), allora comparare le correlazioni fra loro dà probabilmente risultati spuri. La versione di Wallerstein del problema di Galton è che un sistema sovraordinato determina le condizioni interne degli stati nazionali. I teorici del sistemamondo si sono spinti fino a sfidare l'esistenza stessa dell'analisi comparata. Ma la crescente internazionalizzazione del mondo e la corrispondente interdipendenza crescente fra gli stati-nazione solleva interrogativi fondamentali sulla possibilità di comparare correlazioni fra dati di diverse nazioni e suggerisce la necessità di chiamare in causa nuove modalità di ricerca e nuove metodologie per l'analisi comparata. Fra queste vi sono:

- inventare categorie che esprimono qualità relazionali fra unità nazionali e far sì che queste ultime siano le unità fondamentali di analisi anziché le unità nazionali medesime. Parallelamente, affidarsi a qualità relazionali fra individui (per esempio, ruoli, strutture sociali) e trattare queste come unità fondamentali di analisi anziché gli individui.

- Abbandonare l'idea della società o dello stato come unità 
autonoma che forma «relazioni» con altre unità; si dovranno inventare nuovi modi di concettualizzare l'interpretazione delle influenze. La società, la nazione e lo stato dovranno essere caratterizzati come unità che «filtrano» e «condizionano» le influenze anziché come unità che «reagiscono» a tali forze. Il linguaggio dominante dovrà far riferimento alle unità statali quali sistemi con membrane permeabili. Questa alterazione modificherà anche le nostre nozioni di equilibrio e di altri concetti che derivano dalla concezione di sistemi con unità discrete.

- Gran parte della nostra analisi comparata dovrà essere dedicata a livelli di analisi diversi dallo stato. Dovremo dedicarci a comparare regioni economicamente significative (sia subnazionali che sovranazionali), identità culturali solidali che oltrepassano i confini nazionali, movimenti sociali di carattere multinazionale, e così via. In una parola, dovremo essere più consapevoli di come selezioniamo le unità di analisi a scopo di comparazione.

Vorrei concludere su questa nota. Ritengo che la metodologia dell'analisi comparata sia un soggetto vivo, come dev'essere, ma dev'essere anche il soggetto di un esame e una revisione continua alla luce delle condizioni con cui il mondo in mutamento ci confronta.

[Traduzione di Ettore Recchi]

\section{Riferimenti bibliografici}

Armer, M. e A.D. Grimshaw (a cura di) (1973), Comparative Social Research: Methodological Problems and Strategies, New York, Wiley.

Bach, R. (1977), Methods of Analysis in the Study of the World-Economy: A Comment on Rubinson, in «American Sociological Review», 42, 5, pp. 811-842.

Brady, H.E. (1995), Symposium on «Designing Social Inquiry», Part 2, in «Political Methodologist», 6, 2, pp. 11-19.

Caporaso, J.A. (1995), Research Design, Falsification, and the Qualitative-Quantitative Divide, in «American Political Science Review», 89, 1, pp. 3-6.

Frank, A.G. (1971), The Sociology of Underdevelopment and the Underdevelopment of Sociology, London, Pluto Press. 
King, G., R.O. Kehoane e S. Verba (1994), Designing Social Inquiry: Scientific Inference in Qualitative Research, Princeton, Princeton University Press.

Lijphart, A. (1971), Comparative Politics and the Comparative Method, in «American Political Science Review», 65, 3, pp. 682-693.

Lipset, S.M. e N. Smelser (a cura di) (1961), Sociology: The Progress of a Decade, Englewood Cliffs, Prentice-Hall.

McMichael, P. (1990), Incorporating Comparison within a World Historical Perspective: An Alternative Comparative Method, in «American Sociological Review», 55, 3, pp. 385-397.

Przeworski, A. e H. Teune (1970), The Logic of Comparative Social Inquiry, New York, Wiley.

Ragin, C. (1981), Comparative Sociology and the Comparative Method, in «International Journal of Comparative Sociology», 22, 1-2, pp. 102-120.

- (1987), The Comparative Method: Moving Beyond Qualitative and Quantitative Strategies, Berkeley, University of California Press.

Rogowski, R. (1995), The Role of Theory and Anomaly in Social-scientific Inference, in «American Political Science Review», 89, 1, pp. 7-10.

Sartori, G. (1970), Concept Misinformation in Comparative Politics, in «American Political Science Review», 64, 4, pp. 1033-1053.

Skocpol, T. e M. Somers (1980), The Uses of Comparative History in Macrosocial Inquiry, in «Comparative Studies in Society and History», 22, pp. 174-197.

Smelser, N.J. (1967), Notes on the Methodology of the Comparative Analysis of Economic Activity, in Transactions of the Sixth World Congress of Sociology, The Hague, Nauwelarts, vol. II, pp. 7-21.

- (1971), Alexis de Tocqueville as Comparative Analyst, in I. Vallier (a cura di), Comparative Methods in Sociology: Essays on Trends and Applications, Berkeley, University of California Press.

- (1973), The Methodology of Comparative Analysis, in D.P. Warwick e S. Osherson (a cura di), Comparative Research Metho$d s$, Englewood Cliffs, Prentice-Hall.

- (1976), Comparative Methods in the Social Sciences, Englewood Cliffs, Prentice-Hall.

Tilly, C. (1984), Big Structures, Large Processes, Huge Comparisons, Russell Sage Foundation, New York.

Vallier, I. (a cura di) (1971), Comparative Methods in Sociology: Essays on Trends and Applications, Berkeley, University of California Press.

Wallerstein, I. (1974), The Modern World System I: Capitalist Agriculture and the Origins of the European World Economy in the Sixthteen Century, New York, Academic Press.

- (1978), The Capitalist World Economy, Cambridge, Cambridge University Press. 
- (1984), The Politics of the World Economy: The States, the Movements and the Civilizations, Cambridge, Cambridge University Press. 